\title{
Interferometry using adiabatic passage in dilute-gas Bose-Einstein condensates
}

\author{
M. Rab, ${ }^{1}$ A. L. C. Hayward, ${ }^{1}$ J. H. Cole, ${ }^{2}$ A. D. Greentree,,${ }^{1,3}$ and A. M. Martin ${ }^{1}$ \\ ${ }^{1}$ School of Physics, The University of Melbourne, Victoria 3010, Australia \\ ${ }^{2}$ Chemical and Quantum Physics, School of Applied Sciences, RMIT University, Victoria 3001, Australia \\ ${ }^{3}$ Applied Physics, School of Applied Sciences, RMIT University, Victoria 3001, Australia
}

(Received 25 July 2012; published 5 December 2012)

\begin{abstract}
We theoretically examine three-well interferometry in Bose-Einstein condensates (BECs) using adiabatic passage. Specifically, we demonstrate that a fractional coherent transport adiabatic passage protocol enables stable spatial splitting in the presence of nonlinear interactions. A reversal of this protocol produces a coherent recombination of the BEC with a phase-dependent population of the three wells. The effect of nonlinear interactions on the interferometric measurement is quantified and is found to lead to an enhancement in sensitivity for moderate interaction strengths.
\end{abstract}

DOI: 10.1103/PhysRevA.86.063605

PACS number(s): 03.75.Lm, 07.77.-n, 05.30.Jp

\section{INTRODUCTION}

Since the creation of the first Bose-Einstein condensate (BEC) [1,2], cold-atom experiments have provided a powerful platform for the study of macroscopic quantum states $[3,4]$, emulation of solid-state physics $[5,6]$, and insight into manybody quantum phenomena. The utility of BECs stems from the experimental freedom to control many system parameters, including the interaction strength, degrees of freedom, size, and shape of the BEC.

One enticing proposal is to use BECs for interferometry [7-9]. BEC interferometers would have many advantages over their optical counterparts. Trapped-atom interferometers can be sensitive to changes in mass, charge, magnetic moment, and polarizability. As with optical interferometry, a BEC interferometer involves the spatial splitting, followed by the generation of a relative phase difference between split components and then coherent recombination of the quantum state. Performing these operations in a way that is relatively insensitive to small errors in the implementation is a necessary precondition for a reliable interferometer.

The conventional two-well splitting of a BEC is highly sensitive to atom-atom interactions where phase diffusion and Josephson oscillations lead to a loss of phase resolution [10-12]. Beam splitting via laser-induced adiabatic passage has the advantage of being robust to changes in experimental parameters. Marte et al. [13] proposed a variant of the efficient and robust three-state adiabatic process known as stimulated Raman adiabatic passage (STIRAP) [14-17], called fractional STIRAP (f-STIRAP) [18,19]. This scheme can be used to generate any preselected coherent superposition of two atomic states, $|1\rangle$ and $|3\rangle$, via an intermediate excited state, $|2\rangle$. Electromagnetic pulses are used to couple states $|1\rangle$ to $|2\rangle$ and $|2\rangle$ to $|3\rangle$, characterized by coupling parameters $\Omega_{12}$ and $\Omega_{23}$. As in STIRAP, $\Omega_{23}$ precedes $\Omega_{12}$, but unlike STIRAP where $\Omega_{23}$ vanishes first, here, the two pulses vanish simultaneously while maintaining a constant ratio of amplitudes. The ratio of probability amplitudes of the resulting coherent superposition of states $|1\rangle$ and $|3\rangle$ is proportional to the ratio $\Omega_{23} / \Omega_{12}$. Hence, stopping at $\Omega_{23} / \Omega_{12}=1$ can create an atomic beam splitter as demonstrated experimentally by Weitz et al. [20].

Here, we propose an alternative method for the spatial splitting and coherent recombination of a BEC based on the ideas underpinning f-STIRAP. In three-well atomic [21] and electronic quantum dot [22] systems, the coherent spatial transport of single-particle quantum states is known as coherent tunneling adiabatic passage (CTAP). Recent papers have shown that this principle can be extended to interacting manybody quantum systems, such as BECs, both in the quantum [23] and in the semiclassical mean-field limits [24-30].

Analogous to f-STIRAP, fractional CTAP (f-CTAP) allows for the creation of a coherent spatial superposition of eigenstates of wells 1 and 3. For photons, f-CTAP has been demonstrated experimentally in three-channel optical waveguides [31,32]. Consider the initial state with the BEC confined to well 1, schematically shown in Fig. 1(a). The atomic population of the BEC is transported from well 1 to well 3 through adiabatic changes to the tunneling rates between the wells and is halted once the BEC is equally split [see Fig. 1(b)]. At this point, one component of the BEC can be made to interact with some system of interest for time $\tau$. In the case where a phase difference accumulates between the two states of the superposition, this phase can be determined via an interferometric measurement as demonstrated in two-well systems [9,33] by releasing the BEC from the three-well system. In this paper, f-CTAP is proposed to also be used in the recombination stage of the interferometer as reversing the f-CTAP protocol results in a phase-dependent population of the three wells [see Fig. 1(c)]. Interferometric f-CTAP needs to be performed on a time scale that is much longer than nonadiabatic methods. However, the tunneling interaction between the split BECs maintains mutual coherence throughout the splitting and recombination processes. As with CTAP, the f-CTAP protocol has the advantage of being robust to variations in trap parameters and pulse time.

To explore the dynamics of our three-well system, we employ the three-mode Gross-Pitaevskii equation (GPE) [25,26,28,34-36]. In the noninteracting limit, we show analytically that a BEC initially residing in well 1 can be split between wells 1 and 3 and can be recombined to give a phase-dependent density in well 1 [see Fig. 1]. To understand the role of interactions, we use a nonlinear three-mode treatment. The presence of interactions gives rise to a window where canonical CTAP can occur and a regime where more sensitive interferometric sensing can be observed. By solving the corresponding classical equations of motion, we identify 


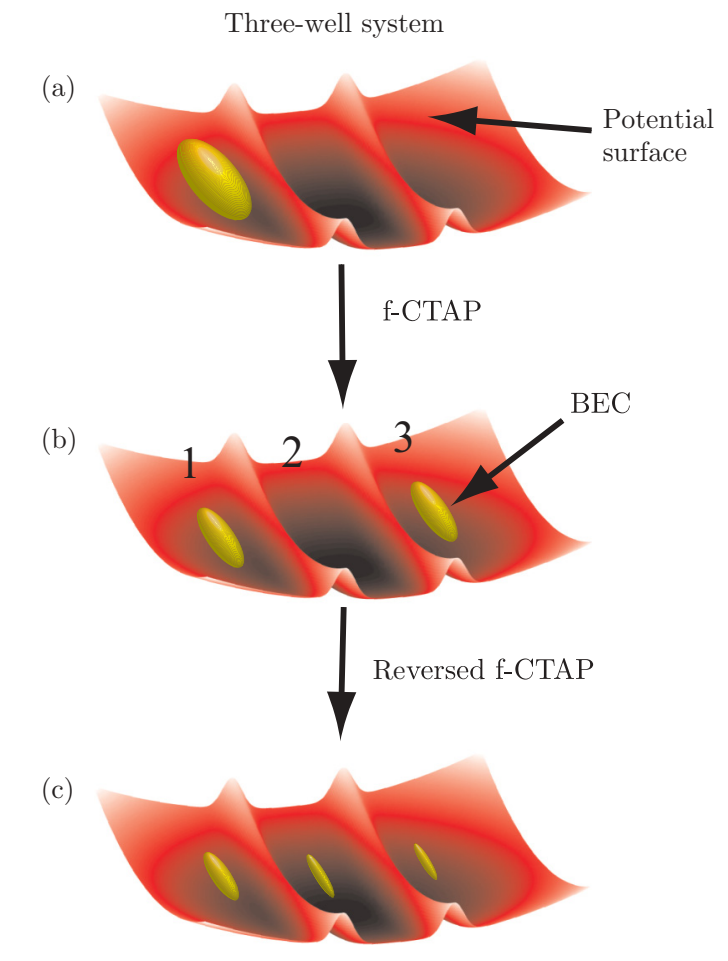

FIG. 1. (Color online) Schematic of a three-well system: at (a) $t=0$, at (b) $t_{p} / 2<t<t_{p} / 2+\tau$ where $\tau$ is the hold time required for phase accumulation, and at (c) $t=t_{p}+\tau$. The system consists of two parallel repulsive Gaussian barriers embedded in an ambient harmonic trap, dividing the system into three wells. At $t=0$ - (a) - the BEC resides in a well. At $t=t_{p} / 2-$ (b)- the BEC is split into two equal components residing in wells 1 and 3. To perform the interferometric measurement, the tunneling rates are then kept constant for some hold time $\tau$ during which a relative phase difference may be accumulated. At $t=t_{p}+\tau$-(c) - the two BECs are recombined, which leads to a phase-dependent population in well 1.

the nonlinear eigenstates of the system, identifying the bifurcation point where extra nonlinear states appear, and investigate the stability of the CTAP state.

The nonlinear dynamics that are obtained from the interaction CTAP model have been mapped directly onto a corresponding three-dimensional GPE [29] showing that the adiabatic transport of a BEC containing $2000{ }^{7} \mathrm{Li}$ atoms can be achieved over $20 \mu \mathrm{m}$ within an ambient harmonic trap of $\omega=2 \pi \times 40 \mathrm{~Hz}$.

\section{NONINTERACTING MODAL APPROXIMATION}

Consider a three-well system. For sufficiently large wells, the system is accurately described by the modal approximation $[14,25,26,28,36,37]$. Each well is approximated by a single mode $\Psi_{j}$, giving

$$
\Psi=\sum_{j=1}^{3} \psi_{j}(t) \Phi_{j}(x),
$$

where the amplitude of each mode $j$ is expressed as $\psi_{j}=$ $\sqrt{N_{j}} e^{i \phi_{j}}$ with $N_{j}$ and $\phi_{j}$ being the modal occupation and phase, respectively. The system is normalized such that $\sum_{j=1}^{3} N_{j}(t)=1$. In this basis, the Hamiltonian of the threewell system is as follows:

$$
H=\hbar \Omega\left(\begin{array}{ccc}
U_{1} & -\Omega_{12} & 0 \\
-\Omega_{12} & U_{2} & -\Omega_{23} \\
0 & -\Omega_{23} & U_{3}
\end{array}\right),
$$

where $\Omega_{i j}$ is the dimensionless tunneling rate between modes $i$ and $j$, determined by the wave-function overlap. The dimensionless on-site interaction energy per particle is $U_{j}=$ $E_{j}^{0}+g N_{j} . \hbar \Omega E_{j}^{0}$ is the ground-state energy of well $j$ with $\Omega$ being the maximum tunneling rate that sets the characteristic energy scale of the system and $g$ as the dimensionless parameter, describing the nonlinear atomic interactions.

We first describe the f-CTAP protocol for BEC splitting and recombination in the noninteracting regime $g=0$.

\section{A. BEC splitting with fractional CTAP}

In the absence of nonlinear atomic interactions $(g=0)$ and for $E_{1}^{0}=E_{3}^{0}=0, E_{2}^{0}=\Delta$, the eigenstates of the Hamiltonian Eq. (2) are as follows:

$$
\begin{gathered}
D_{0}=\frac{\Omega_{23} \Phi_{1}}{\sqrt{\Omega_{23}^{2}+\Omega_{12}^{2}}}-\frac{\Omega_{12} \Phi_{3}}{\sqrt{\Omega_{23}^{2}+\Omega_{12}^{2}}} \\
D_{ \pm}=\frac{1}{\sqrt{\gamma(\gamma \pm \Delta)}}\left[\sqrt{2} \Omega_{12} \Phi_{1}-\frac{\Delta \pm \gamma}{\sqrt{2}} \Phi_{2}+\sqrt{2} \Omega_{23} \Phi_{3}\right]
\end{gathered}
$$

where $\gamma=\sqrt{\Delta^{2}+4\left(\Omega_{12}^{2}+\Omega_{23}^{2}\right)}$. The $D_{0}$ eigenstate has no population in the center well for all $\Delta$ and, in the limit of $\Omega_{12} / \Omega_{23} \ll 1$, the atomic population is confined entirely to well 1.

Consider the scenario where the BEC initially resides in well $1\left(\Omega_{12} / \Omega_{23}=0\right)$. Adiabatic transport through the $D_{0}$ state such that the BEC is equally split between wells 1 and at $t=t_{p} / 2$ requires a smooth transformation of $\Omega_{12}=0$ to $\Omega_{12}=\Omega_{23}=1 / 2$. A pulsing sequence for $\tau=0$ that meets this criterion is as follows:

$$
\begin{gathered}
\Omega_{12}(t)=\frac{1}{2} \sin ^{2}\left[\pi t / t_{p}\right], \\
\Omega_{23}(t)=\frac{1}{2}+\frac{1}{2} \cos ^{2}\left[\pi t / t_{p}\right],
\end{gathered}
$$

as shown in Fig. 2(a). The mode energies for this pulse sequence are shown in Fig. 2(b) with the population of the three wells shown in Fig. 2(c) (where adiabatic pulsing has been assumed). At $t_{p} / 2, N_{1}=N_{3}=0.5$, which is the condition we require for sensing.

The robustness of the splitting protocol relies on adiabatic changes to the system tunneling. For noninteracting BECs, the adiabaticity is quantified by the adiabaticity parameter,

$$
\alpha=\max _{i= \pm} \frac{\left|\left\langle D_{0} ; t\left|\frac{\partial}{\partial t} H(t)\right| D_{i} ; t\right\rangle\right|}{\left|E_{0}(t)-E_{i}(t)\right|^{2}},
$$

which is shown in Fig. 2(d) through the protocol. Adiabatic transfer requires $t_{p}$ to be chosen such that $\alpha \ll 1$ over the entirety of the protocol. 
(a)
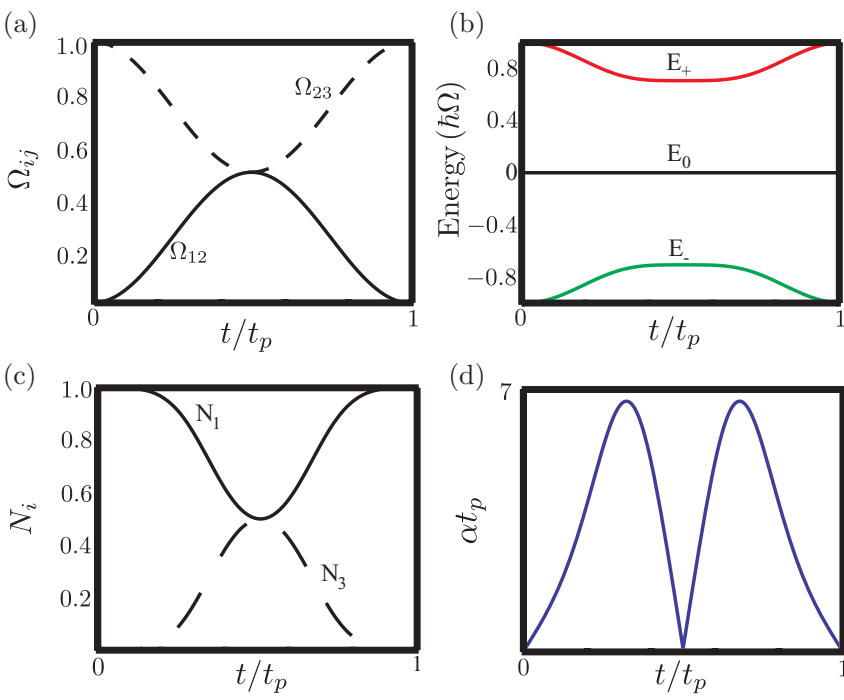

FIG. 2. (Color online) Ideal three-well system with $\Delta=0$ and $g=0$ : (a) Proposed pulsing scheme: Eqs. (5) and (6). (b) Energies of the eigenmodes: $D_{0}$ and $D_{ \pm}$. (c) Evolution of the occupation of the three wells: solid curve: $N_{1}$ and dashed curve: $N_{3}$. (d) Adiabaticity parameter $\alpha t_{p}$. In these figures, we have assumed that $\tau=0$.

\section{B. Phase interferometry with fractional CTAP}

Once the BEC is split, interaction with a target system can induce a relative phase difference between the two components. Since $\Omega_{12}=\Omega_{23}=1 / 2$ at $t=t_{p} / 2$, phase information is shared between the BECs in wells 1 and 3 . This enables the mutual coherence between the two BECs during splitting to be maintained, inhibiting phase diffusion. To enable the desired phase accumulation, tunneling between wells 1 and 3 needs to be suppressed. This can be achieved via a variety of means, such as (i) applying a blocking laser field at the middle point of well 2 or (ii) reducing $\Omega_{12}$ and $\Omega_{23}$ adiabatically to zero. After the decoupling of the BECs, phase diffusion will occur. The BECs can be recoupled some time later by, for example, (i) switching off the blocking laser field or (ii) adiabatically increasing $\Omega_{12}$ and $\Omega_{23}$ to $1 / 2$. We assume that the process of decoupling and recoupling occurs over time scale $\tau$. Considering the case where the two BECs accumulate a phase difference $\phi$ over $\tau$, the state of the system is then

$$
\Psi_{S}\left(t_{p} / 2+\tau, \phi\right)=\frac{1}{\sqrt{2}}\left(\Phi_{1}-e^{i \phi} \Phi_{3}\right) .
$$

The acquired phase difference during $\tau$ moves the system out of the $D_{0}$ state and into a superposition of all three eigenstates. Hence, the resulting change in eigenstate populations for a given $\phi$ is as follows:

$$
\begin{gathered}
N\left(D_{+}\right)=\sin ^{2}(\phi / 2) \sin ^{2} \theta, \\
N\left(D_{-}\right)=\sin ^{2}(\phi / 2) \cos ^{2} \theta, \\
N\left(D_{0}\right)=\cos ^{2}(\phi / 2),
\end{gathered}
$$

where

$$
\theta=\frac{1}{2} \arctan [\sqrt{2} / \Delta]
$$

Adiabatic evolution of the system through to $t=t_{p}+\tau$ returns the barriers back to their initial configuration where
$\Omega_{12}=0$ and $\Omega_{23}=1$. At the end of the splitting and phase accumulation $\left(t=t_{p}+\tau\right)$, the $D_{0}$ eigenstate is simply the state in well 1 with the final population given by

$$
N_{1}\left(t_{p}+\tau\right)=N\left(D_{0}\right)=\cos ^{2}(\phi / 2) \text {. }
$$

Thus, the phase difference accumulated during $\tau$ manifests as a population imbalance at the end of the protocol. Density measurements on the final state of the BEC then allow determination of the phase $\phi$. This is demonstrated by full numerical integration of the three-mode GPE in Sec. IIIC where the $g=0$ lines in Fig. 9 confirm the phase response predicted by Eq. (13).

Noise in the tunneling throughout the transport can be suppressed by considering an experimental setup where a single laser is split proportionally between two barriers to mediate the tunneling interaction. Hence, noise in $\Omega_{12}$ and $\Omega_{23}$ due to fluctuations in the intensity of the source laser will be coupled. As shown in Eckert et al. [21], adiabatic transport is still achievable in the presence of such noise.

Any small asymmetry in the final tunneling terms of the Hamiltonian results in a loss of sensitivity proportional to the population difference between wells $|1\rangle$ and $|3\rangle$ and as such, does not affect our analysis.

\section{NONLINEAR THREE-MODE APPROXIMATION}

Controllable nonlinearities are one of the defining features of BEC dynamics as compared to analogous photonic systems. Accordingly, we now consider the effect of a nonzero $g$ on the f-CTAP interferometric protocol. Interparticle interaction in BECs gives rise to a raft of interesting phenomena, for example, quantum phase transitions [6], solitons [38-41], and entanglement generation [42]. However, in the context of BEC interferometry, these interactions are, in general, problematic as they induce phase diffusion. These interactions manifest as a nonlinearity in the mean-field description, leading to highly nontrivial dynamics. We analyze the behavior of the system by mapping the nonlinear GPE to a classical Hamiltonian. The nature of the stationary states in the classical model determines the behavior of the protocol in the adiabatic limit. This analysis is complimented by numerical simulation. Simulation of the protocol confirms the conclusions of the classical analysis in the large $t_{p}$ limit and reveals several interesting phenomena for finite $t_{p}$. Interestingly, we find that the presence of nonlinearities can lead to an enhanced phase sensitivity.

\section{A. Mapping to classical variables}

The presence of a nonlinearity in the GPE makes analysis of the dynamics significantly more difficult. Notably, the superposition principle is no longer valid, making an eigenstate decomposition impossible. In general, there will be more eigenstates than the Hilbert space dimension. The system can be approached through concepts borrowed from classical mechanics as there is a mapping of our system to a classical Hamiltonian. From this perspective, we study the stationary points in the classical phase space, that correspond to the eigenstates of the original system. 
Any quantum system with Hilbert space dimension $N$ can be mapped onto a classical system with $2 N$ degrees of freedom, namely, the $N$ phases and $N$ amplitudes of the state vector. Symmetry under global phase shifts leads to a conservation of probability amplitude as an integral of motion, and if the Hamiltonian is time independent, the total energy also is an integral of motion. This implies that two-mode BECs have nonchaotic dynamics. In the three-well case, integrable dynamics are no longer guaranteed. The presence of chaotic dynamics has implications for the splitting and recombination elements of the interferometer as adiabatic transport through a chaotic region of phase space is not possible. Linear quantum systems map onto $N$-dimensional harmonic oscillators and are necessarily integrable. Introduction of nonlinear terms leads to more complicated classical dynamics, which in some cases, can be chaotic. This is indeed the case for the three-well BEC $[43,44]$.

We take the classical degrees of freedom to be the amplitude squared and phase of the BEC in each well: $N_{i}=\left|\psi_{i}\right|^{2}, \phi_{i}=$ $\arg \left(\psi_{i}\right)$. The number of degrees of freedom can be reduced by 2 using conservation of probability and global phase symmetry,

$$
N_{2}=1-N_{1}-N_{3}, \quad \phi_{12}=\phi_{1}-\phi_{2}, \quad \phi_{32}=\phi_{3}-\phi_{2} .
$$

The classical Hamiltonian is then

$$
\begin{aligned}
H / \hbar \Omega= & \Delta\left(1-N_{1}-N_{3}\right)+\frac{g}{2}\left[N_{1}^{2}+N_{3}^{2}+\left(1-N_{1}-N_{3}\right)^{2}\right] \\
& -2 \Omega_{12} \sqrt{N_{1}\left(1-N_{1}-N_{3}\right)} \cos \phi_{12} \\
& -2 \Omega_{23} \sqrt{N_{3}\left(1-N_{1}-N_{3}\right)} \cos \phi_{32},
\end{aligned}
$$

and the equations of motion are as follows:

$$
\begin{aligned}
& \dot{\phi_{12} / \hbar \Omega}=-\frac{\Omega_{12}\left(2 N_{1}+N_{3}-1\right) \cos \phi_{12}}{\sqrt{N_{1}\left(1-N_{1}-N_{3}\right)}}-\Delta \\
& -\frac{\Omega_{23} N_{3} \cos \phi_{32}}{\sqrt{N_{3}\left(1-N_{1}-N_{3}\right)}}+g\left(2 N_{1}+N_{3}-1\right), \\
& \dot{N}_{1} / \hbar \Omega=2 \Omega_{12} \sqrt{N_{1}\left(1-N_{1}-N_{3}\right)} \sin \phi_{12} \text {, } \\
& \dot{\phi_{32}} / \hbar \Omega=-\frac{\Omega_{23}\left(N_{1}+2 N_{3}-1\right) \cos \phi_{32}}{\sqrt{N_{3}\left(1-N_{1}-N_{2}\right)}}-\Delta \\
& -\frac{\Omega_{12} N_{1} \cos \phi_{12}}{\sqrt{N_{1}\left(1-N_{1}-N_{3}\right)}}+g\left(N_{1}+2 N_{3}-1\right), \\
& \dot{N}_{3} / \hbar \Omega=2 \Omega_{23} \sqrt{N_{3}\left(1-N_{1}-N_{3}\right)} \sin \phi_{32} .
\end{aligned}
$$

\section{Stationary states}

CTAP, by definition, requires evolution along particular eigenstates. In Sec. II B, we were able to understand sensing by the accumulated phase shifting of the BEC into a superposition of all three eigenstates. However, the nonlinearities in the modal BEC approach invalidate the superposition principle. Nevertheless, studying the states of the system still yields useful insight into the structure of the resulting phase space.

The eigenvalues are given by the stationary solutions to Eq. (19). To distinguish from the $g=0$ case, we denote these stationary states by $D^{\prime}$ with the $D_{0}^{\prime}$ coinciding with the $D_{0}$ split state at $t=t_{p} / 2$,

$$
D_{0}^{\prime}\left(t_{p} / 2\right)=\frac{1}{\sqrt{2}}\left(\Phi_{1}-\Phi_{3}\right)=\Psi_{50: 50} .
$$
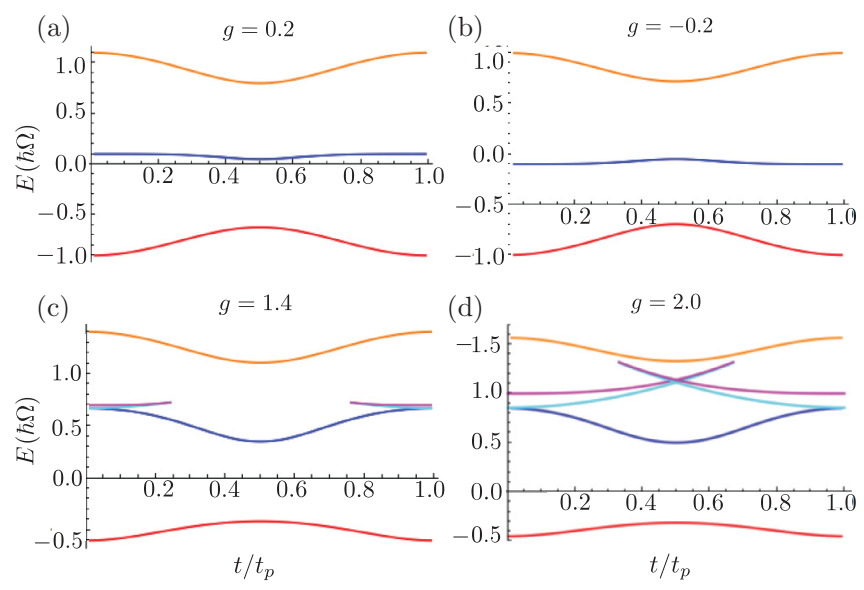

FIG. 3. (Color online) Eigenenergies for a range of interaction strengths with $\Delta=0.1$ and $t_{p} \rightarrow \infty$. As the interaction strength is increased, new eigenstates appear. When $|g| \approx 1.0$, the $D_{0}^{\prime}$ state at $t=0$ is no longer $\psi_{1}$, precluding the possibility of a 50:50 split.

As mentioned above, for the interacting case, the number of eigenvalues is no longer limited to 3 (see Ref. [45] for an indepth discussion). Figure 3 shows the eigenvalues for various values of $g$, using the pulsing sequence defined by Eqs. (5) and (6). At higher interaction strengths, new eigenstates appear. The appearance of these new states marks a bifurcation near the $t=\left\{0, t_{p}\right\}$ limits, which disconnects the $D_{0}^{\prime}$ state from the fully occupied states at the end points. Once these eigenstates appear at $g=g_{c}=\Delta / 2 \pm \sqrt{1+\Delta^{2} / 4}, 50: 50$ splitting is precluded, even in principle.

The appearance of these additional nonlinear eigenstates is a ubiquitous feature of nonlinear systems. However, their effect on the dynamics depends on the pulse sequence that is employed. In particular, Graefe et al. [28] showed that the extra eigenstates are a permanent feature of the eigenspectrum for all $g \Delta \leqslant 0$ and $|g|>|\Delta|$ using a Gaussian pulsing protocol, precluding adiabatic transport. Conversely, $\Omega_{23} \neq 0$, at the start of the protocol as used here, has the effect of suppressing the emergence of these extra nonlinear eigenstates for low values of $|g|$ as seen in the window of robust evolution in Fig. 6. This robustness is further considered in the stability analysis below.

From Eq. (19), it is possible to calculate the wave-function amplitudes in the three wells as a function of the tunneling rates and nonlinear interaction. Figure 4 shows that, for $g=0, \psi_{2}=0$ for all $t$. However, upon the introduction of interactions, $\psi_{2}$ will, in general, be nonzero. After the appearance of the self-trapped mode, the 50:50 split state at $t / t_{p}=0.5$ is no longer adiabatically connected to the fully occupied well 1 state at $t / t_{p}=0$.

\section{Stability}

We now consider the stability of the $D_{0}^{\prime}$ state in the regime of $|g|<\left|g_{c}\right|$. A stationary point is unstable if small perturbations lead to large deviations from the point. These deviations make adiabatic transport of the system impossible as keeping the system near $D_{0}^{\prime}$ requires that $t_{p} \rightarrow \infty$.

When $|g|<\left|g_{c}\right|$, there are just the three eigenstates. However, these differ from the noninteracting case, i.e., the $D_{0}^{\prime}$ 


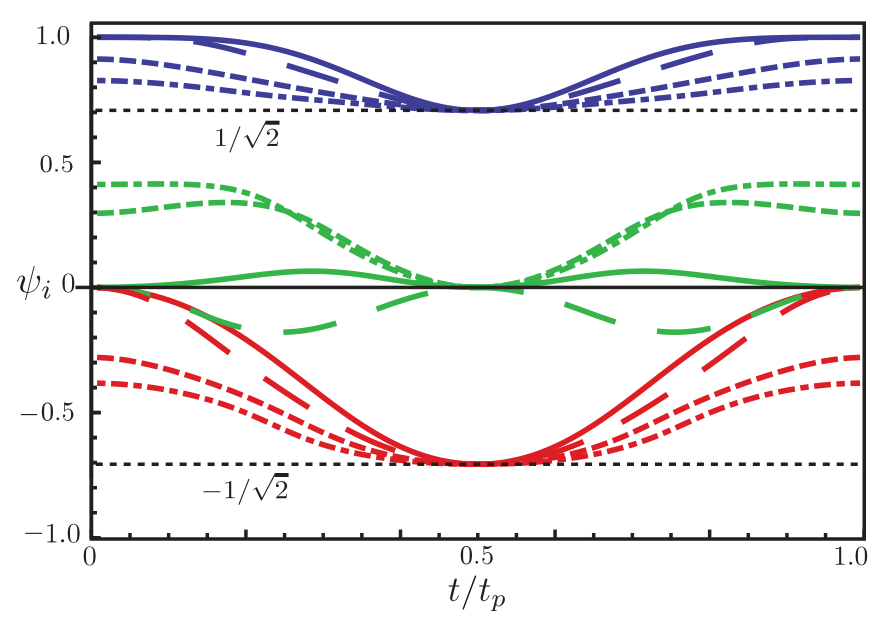

FIG. 4. (Color online) Wave-function components for the $D_{0}^{\prime}$ state with nonzero $g, \Delta=0.1$, and $t_{p} \rightarrow \infty$. Upper (blue): $\psi_{1}$; middle (green): $\psi_{2}$; and lower (red): $\psi_{3}$. Solid curves: $g=0.2$; long dashed curves: $g=-0.6$; short dashed curves: $g=1.4$; and dot-dashed curves: $g=2.0$. Interactions lead to occupation of the middle well during transport as can be seen for $g=0.7$ and $g=1.0$. Note: $\psi_{i}$ is real.

state's eigenenergy is no longer zero and, in general, $\psi_{2}(t) \neq 0$, as demonstrated in Fig. 4. The dynamics in the neighborhood of stationary states can be studied by performing a linear expansion of the equations of motion. Solutions are classified by eigenvalues of the Jacobian at the stationary point,

$$
J_{i j}=\frac{\partial \dot{x}_{i}}{\partial x_{j}},
$$

where the $x_{i}$ 's are the system variables. If the eigenvalues are purely imaginary, then the solutions are oscillatory around the stationary point. An eigenvalue with a positive real part implies the presence of a hyperbolic orbit around the eigenstate, which is unstable.

Figure 5 shows how the stability of the $D_{0}^{\prime}$ state changes along the pulse sequence for $-1<g<1$ for (a) $\Delta=0.0$ and (b) $\Delta=0.1$. For $\Delta=0$, the $D_{0}^{\prime}$ state is strictly only stable for $g=0$ throughout the pulse sequence. However, the introduction of $\Delta$ stabilizes the $D_{0}^{\prime}$ state throughout the transport protocol. Specifically, for $\Delta=0.1$, transport is stable in the range of $|g| \lesssim 0.2$, and as can be seen in Fig. 4, complete
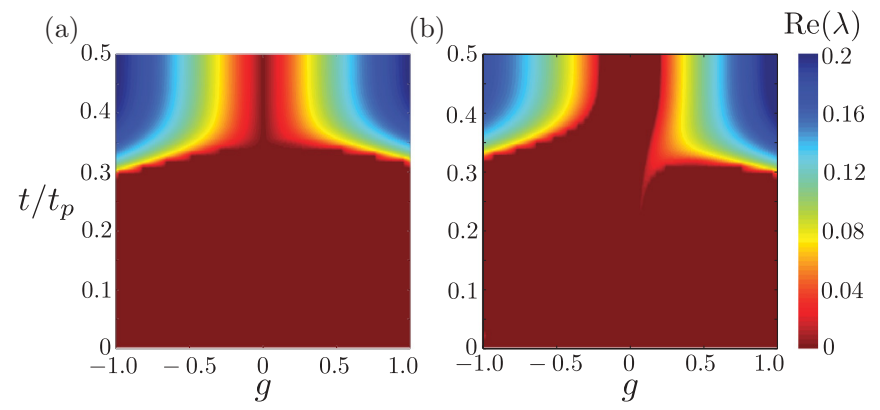

FIG. 5. (Color online) Real part of the eigenvalue of the Jacobian at the $D_{0}^{\prime}$ state. (a) $\Delta=0.0$. (b) $\Delta=0.1$. Transport through regions with $\operatorname{Re}(\lambda)=0$ allows complete fidelity for the protocol. For $\Delta=0$, the $D_{0}^{\prime}$ state is unstable for all $g \neq 0$, albeit with small $\operatorname{Re}(\lambda)$. fidelity for transport to a split state is possible for extended pulse times. At higher $|g|$, transporting through regions of instability leads to a loss of fidelity. For an intermediate interaction strength, this instability is small, and it is possible to find a balance between the adiabaticity requirement and the slow divergence of the $D_{0}^{\prime}$ state, which still allows high fidelity for the splitting protocol. Hence, we have identified $\Delta=0.1$ as advantageous to stable transport. We adopt this parameter in the following analysis.

\section{B. Nonlinear fidelity of splitting}

We now explore the robustness of the f-CTAP splitting protocol by direct numerical evolution of the nonlinear Hamiltonian. Specifically, we solve for $\psi_{j}$ in the presence of nonlinear interactions and finite pulsing times via

$$
\frac{i}{\Omega} \frac{\partial}{\partial t} \Psi=H \Psi .
$$

We are interested in the transportation of the BEC to the split state. We, therefore, define the fidelity as the overlap between the $\Psi_{50: 50}$ state and the transported state $\Psi\left(t_{p} / 2\right)$ as determined from Eq. (22),

$$
\epsilon=\left|\left\langle\Psi_{50: 50} \mid \Psi\left(t_{p} / 2\right)\right\rangle\right|^{2} .
$$

Figure 6 shows the fidelity of BEC splitting in the presence of interactions. Qualitatively, for small values of $|g|$, full adiabatic transport is still possible for $t_{p} / 2>10 \Omega^{-1}$. However, larger interaction strengths lead to oscillations and complete loss of fidelity in the splitting procedure.

The regions of efficient splitting in Fig. 6 can be explained in terms of the stability of the $D_{0}^{\prime}$ state (Fig. 5). For the pulsing scheme given by Eqs. (5) and (6), stable transport to the split state is achieved for $|g|<0.2$ in the adiabatic limit (long dashed lines in Fig. 6). This region is characterized by its close-to-linear behavior where an increase in total pulse time leads to a corresponding increase in fidelity. The small instability that exists for positive $g$ slightly reduces the fidelity of transport for long pulse times. The absence of extra nonlinear states (Fig. 3) and stability of the $D_{0}^{\prime}$ state in this range (Fig. 5) means that the fidelity of

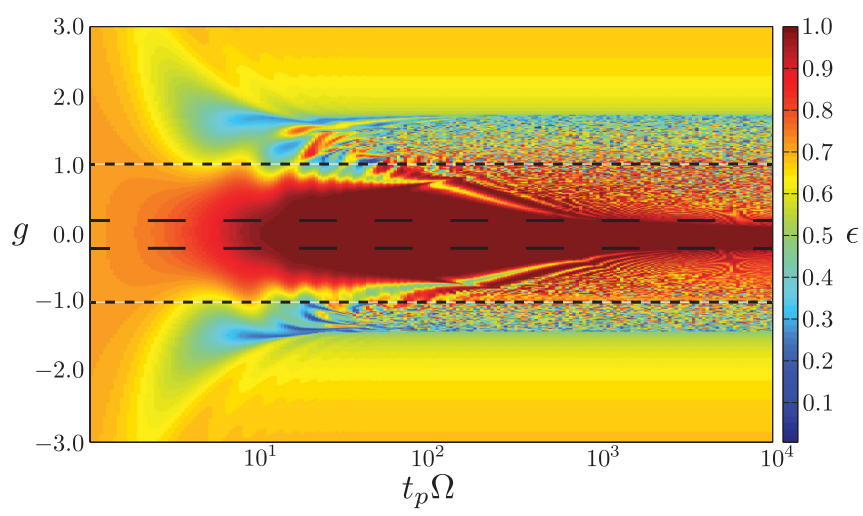

FIG. 6. (Color online) Fidelity $\epsilon$ of split as defined in Eq. (23) as a function of pulse time $t_{p}$ and interaction strength $g$ at $t=t_{p} / 2$ with $\Delta=0.1$. Regions of good fidelity $\epsilon \approx 1$ are denoted by dark red. The short dashed lines denote $g=g_{c}$ where the bifurcation appears, and the long dashed lines denote $|g|=0.2$. 

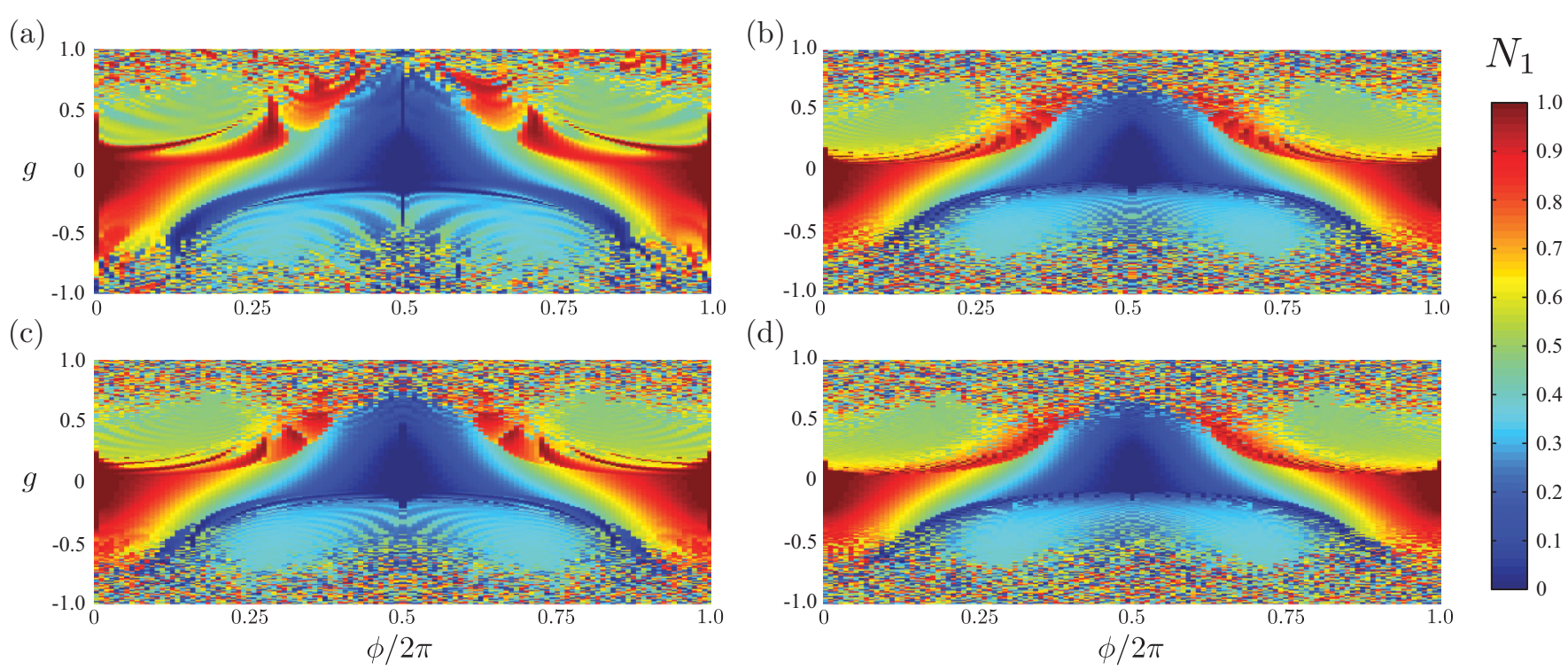

FIG. 7. (Color online) Occupation of well 1 after ideal BEC splitting and recombination using reversal of f-CTAP protocol for (a) $t_{p}=200 \Omega^{-1}$, (b) $t_{p}=500 \Omega^{-1}$, (c) $t_{p}=1000 \Omega^{-1}$, and (d) $t_{p}=2000 \Omega^{-1}$ with $\Delta=0.1$ as a function of the phase difference $(\phi)$ between wells 1 and 3 at $t=t_{p} / 2$ and the strength of the interatomic interactions $|g| \leqslant 1$.

transport obeys the linear adiabatic law up to very long pulse times.

For $|g|>0.2$, transport for the $D_{0}^{\prime}$ state becomes unstable (Fig. 5) with the growth of this instability increasing with $|g|$. To achieve good transport in this regime, the optimal pulse time must be found. This comes from competition between the adiabaticity of the transport protocol and the instability time scale of the $D_{0}^{\prime}$ state with shorter $t_{p} / 2$ being favored for stronger interactions. At $g=g_{c}$, the appearance of extra nonlinear states near the $D_{0}^{\prime}$ state, which only extend partially along the pulsing scheme as shown in Fig. 3, prevent stable splitting for any $t_{p} / 2$. Once these extra states extend to $t=t_{p} / 2$, stability in transport is restored. However, the final state does not overlap the $\Psi_{50: 50}$ state.

The evolution shown in Fig. 6 is the first stage of this interferometric process, and reversal of the protocol, for recombination, naturally leads to high-fidelity transport analogous to full CTAP transport. The full sensing protocol is the subject of the next section.

\section{Phase interferometry in the presence of interactions}

As shown in Sec. II B, the reversal of the f-CTAP splitting process gives a phase-sensitive population in well 1, Eq. (11). Here, we quantify the effect of interactions on this phase measurement by numerically integrating Eq. (22) to determine the population of well 1 at the end of the protocol $N_{1}\left(t_{p}+\tau\right)$. We assume an ideal splitting with $N_{1}\left(t_{p} / 2+\tau\right)=N_{3}\left(t_{p} / 2+\right.$ $\tau)=0.5, E_{1}^{0}=E_{3}^{0}=0$, and $E_{2}^{0}=\Delta=0.1$ in Eq. (2) and allow the BECs to accrue an initial relative phase difference of $\phi$ as in Eq. (8). This investigation of the phase response focuses on the behavior for time scales longer than the linear adiabatic pulse time where the transport is well behaved. Shorter recombination time scales result in a significant loss in sensitivity and are not pertinent to the current investigation.
The final population of well 1 for $t_{p}=200 \Omega^{-1}, t_{p}=$ $500 \Omega^{-1}, t_{p}=1000 \Omega^{-1}$, and $t_{p}=2000 \Omega^{-1}$ (Fig. 7) exhibit large variations that prevent a robust phase measurement for $|g| \gtrsim\left|g_{c}\right|$. For $|g|<0.2$, the phase response is similar to the noninteracting limit [Eq. (11)] and coincides with the region of stability for the $D_{0}^{\prime}$ state (Fig. 5).

We have computed first-order corrections for the periodic orbits using classical perturbation theory [46]. For small $|g|$, the deviation in the final population well 1 from the noninteracting case $\Delta N_{1}$ is given by

$$
\Delta N_{1}=N_{1}-N_{1}(g=0)=\frac{-g \Omega^{2} \sin ^{2}(\phi)}{4 \Delta\left(2+\Delta^{2}\right)},
$$

which agrees with the numerical simulations in Fig. 8. The nonzero $\Delta N_{1}$ implies an increased phase sensitivity due to the interactions, which is robust with respect to the pulse time.

Consistent with Eq. (24), we numerically find that $\Delta N_{1}$ is zero for $\phi=\{0, \pi\}$ (Fig. 8). For long pulse times, Fig. 8(b), the longer time spent traversing the unstable regions of phase space leads to a larger deviation near $\phi=\{0, \pi\}$. For small attractive interactions, Fig. 8 shows that, for $\phi \approx 0$, the interferometer is stable. Conversely, for small repulsive interactions, Fig. 8 shows that, for $\phi \approx \pi$, the interferometer is stable.

Even though bifurcations do not emerge until $|g|>$ $\left|g_{c}\right| \approx 1.0$, Fig. 7 demonstrates that, for interaction strengths $|g|>0.5$, the instability of the symmetric and antisymmetric stationary states at $\phi=\{0, \pi\}$ leads to large variations in density. Large density fluctuations reduce the possibility of recovering any phase information across all of $\phi$ for $g>0.7$.

In Fig. 9, we show the regions where phase-sensitive measurements can be performed in the presence of nonlinear interactions, i.e., $|g| \lesssim 0.35$. Since nonlinear atomatom interactions can introduce unwanted density-dependent fluctuations, which compromise phase sensitivity, atomic 

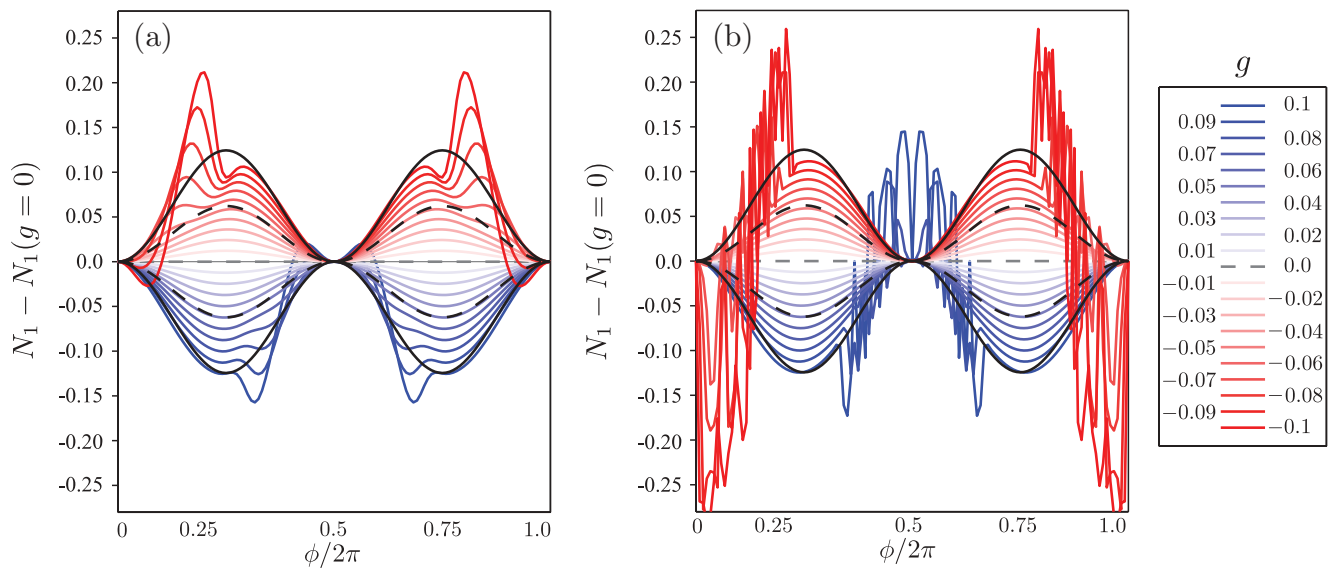

FIG. 8. (Color online) Deviation in the population of well 1 from the noninteracting expected value $N_{1}(|g| \leqslant 0.1)-N_{1}(g=0)$ as a function of the phase difference $(\phi)$ between wells 1 and 3 at $t=t_{p} / 2$ for (a) $t_{p}=100 \Omega^{-1}$ and (b) $t_{p}=2000 \Omega^{-1}$ where $N_{1}(g=0)=\cos ^{2}(\phi / 2)$. The classical first-order perturbation, Eq. (24), is plotted for dashed curves: $|g|=0.05$ and solid black curves: $|g|=0.1$.

interferometry experiments often reduce these interactions via a Feshbach resonance $[47,48]$.

In the small interaction limit with $|g|<0.1$, a robust phasesensitive measurement of $N_{1}$ can be performed by accounting for the correction given by Eq. (24). In this regime, Fig. 9 shows a smooth response of $N_{1}$ with respect to phase with deviations for the noninteracting result shown in Fig. 8. The instability of the $\phi=\pi$ state for attractive interactions, seen as a deviation from the first-order correction in Fig. 8, does decrease the pulse-time sensitivity relative to the stable regions. However, the f-CTAP interferometer should be robust for a broader range of interactions. (a)

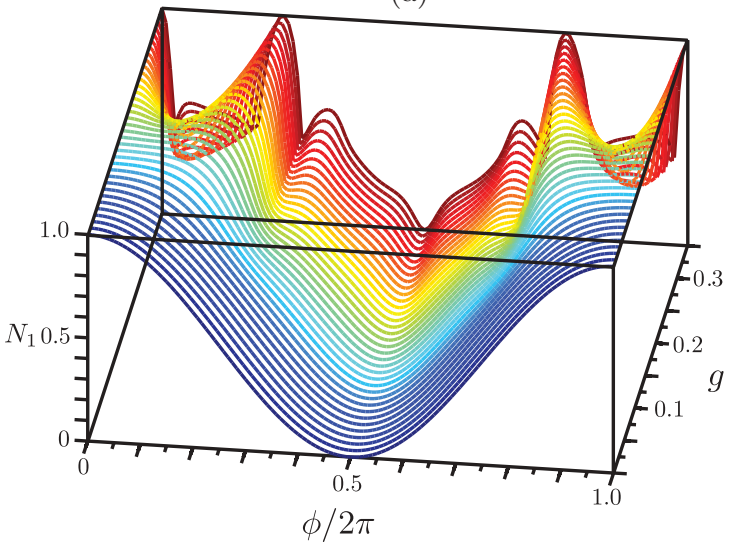

(c)

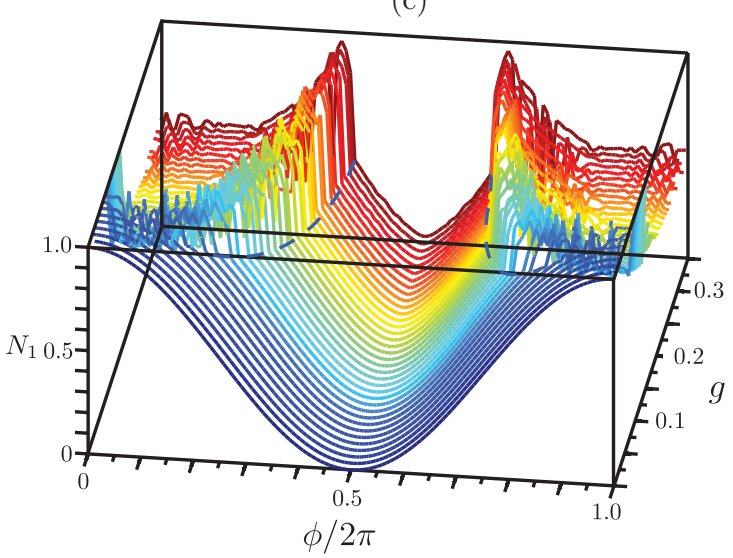

(b)

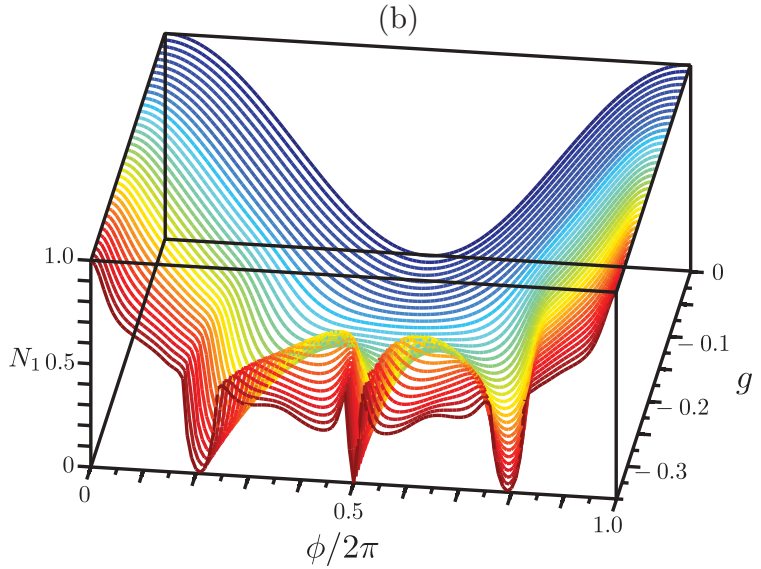

(d)

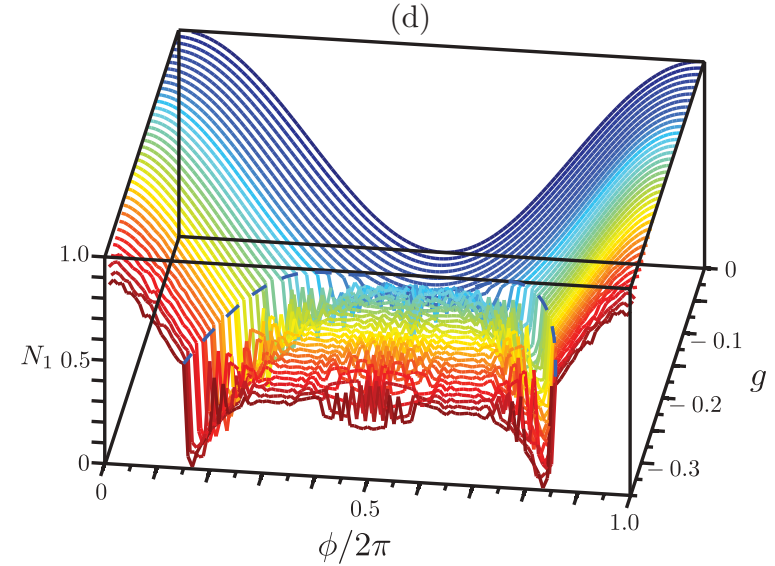

FIG. 9. (Color online) Phase-sensitive measurement of the occupation of well $1 N_{1}\left(t_{p}\right)$ for interatomic interaction strengths $|g| \leqslant 0.35$ after recombination times of (a) and (b) $t_{p}=100 \Omega^{-1}$ and (c) and (d) $t_{p}=3000 \Omega^{-1}$. Ideal splitting of a BEC as defined in Eq. (8) is assumed. 


\section{Enhanced phase sensing}

Here, we highlight how a f-CTAP-based interferometer has two distinct regimes of enhanced phase-sensitive operation, facilitated by control of the interaction strength via a Feshbach resonance. Regime 1 is characterized by sensing around $\phi=$ $\{0, \pi\}$ for short pulse times, whereas, regime 2 requires much longer pulse times but allows for tunable sensing.

In regime 1: For shorter pulse times [see Figs. 7(a) and 7(b)] around $\phi=\{0, \pi\}$, an enhanced phase-sensitive measurement of $N_{1}$ can be performed. For small $|g|$, the first-order corrections to the final population in well 1 [Eq. (24)] are small, and there is very little sensing enhancement. However, for greater $|g|$, large but stable oscillations appear in these regions, leading to enhanced sensing. This occurs for $0.2<$ $g<0.6(\phi \approx 0)$ and $-0.2>g>-0.6(\phi \approx \pi)$ [Fig. 7(a)]. The emergence of these oscillations can be seen in Fig. 8(a) where $\phi=\{0, \pi\}$ for $g<0$ and $g>0$, respectively. As can be seen in Figs. 7(a)-7(d), the range of interaction strengths where the $\phi=\{0, \pi\}$ states return to $N_{1}=\{1,0\}$ reduces with increasing pulse times.

In regime 2: A highly enhanced phase-sensitive measurement of $N_{1}$ can be performed at the point indicated by the dashed line in Figs. 9(c) and 9(d). This stems from the large gradient in the population phase response in the vicinity of the dashed lines. The position of this boundary could be controlled by tuning the interaction strength via a Feshbach resonance. This would, for instance, allow the position of maximum sensitivity for a given phase to be changed depending on the application. This opens up the possibility for using a feedbackbased phase measurement to ensure maximum sensitivity to particular phase changes.
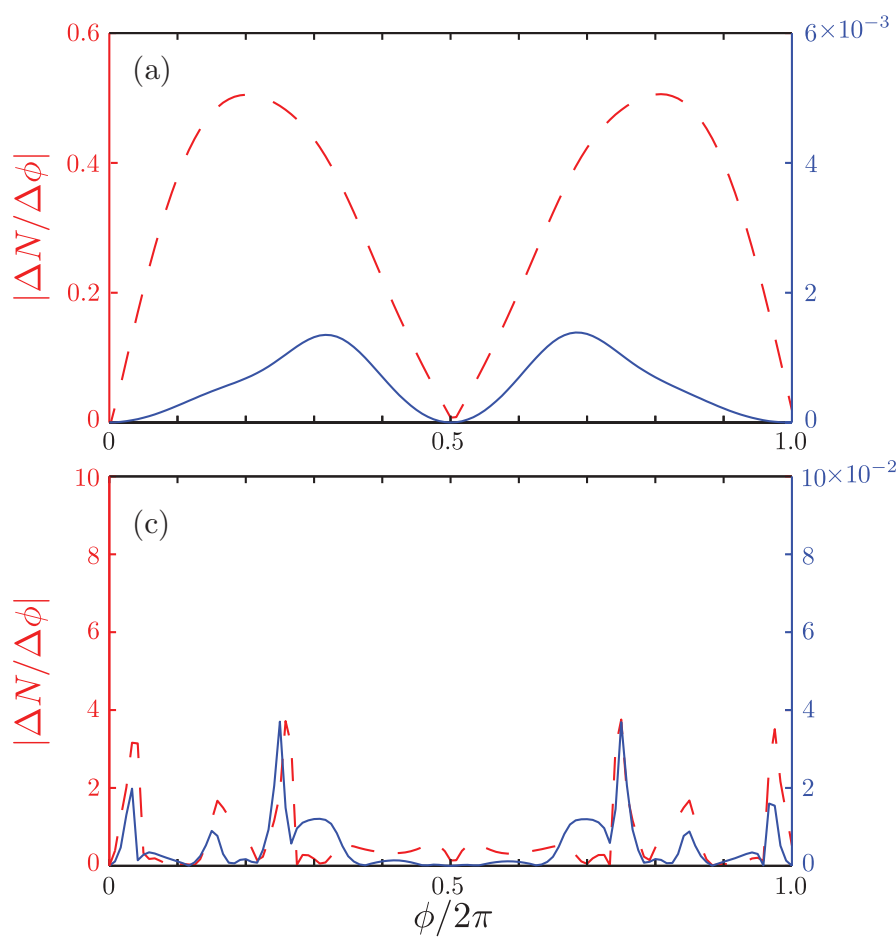

\section{Robustness}

As shown in Sec. III A2, the adiabaticity criteria, Eq. (7), is invalidated by the introduction of the nonlinear interaction term. Hence, here, we associate a robust phase-sensitive measurement with a high sensitivity to changes in relative phase $\partial N / \partial \phi$ and a low sensitivity to changes in pulse time $\partial N / \partial t_{p}$.

For the small interaction limit where the divergence due to the instability is relatively slow, Figs. 10(a) and 10(b) show that, as we increase the pulse time from $t_{p}=100 \Omega^{-1}$ to $t_{p}=2000 \Omega^{-1}$, the pulse-time sensitivity decreases by a factor of $10^{-4}$. However, this deviation is small with respect to phase sensitivity. Although this regime exhibits minimal enhancement of phase sensitivity, it has the robustness and stability of a noninteracting f-CTAP interferometer.

In regime 1: There is some reduction in pulse-time sensitivity relative to the small interaction limit [Fig. 10], however, the pulse-time sensitivity is still a factor of 10 less than the phase sensitivity for $t_{p}=100 \Omega^{-1}$ and is further reduced by a factor of $10^{3}$ for $t_{p}=2000 \Omega^{-1}$ [Figs. 10(c) and 10(d)]. The nonlinear interaction also leads to a smaller enhancement of the phase measurement for $g<0.3$ and $g>0.3$ for $\phi=\{0, \pi\}$, which is more robust to changes in pulse time due to the reduced instability. Despite this, Fig. 10(d) shows that the phase enhancement persists for longer pulse times.

In regime 2: Surprisingly, the robustness of sensing for this range of phase shifts is increased for longer pulse times where peaks in phase sensitivity no longer align with peaks in pulsetime sensitivity [Fig. 10(d)]. The presence of regions in the parameter space, that are insensitive to total pulse time, implies that robust interferometry is possible for the interacting BECs.
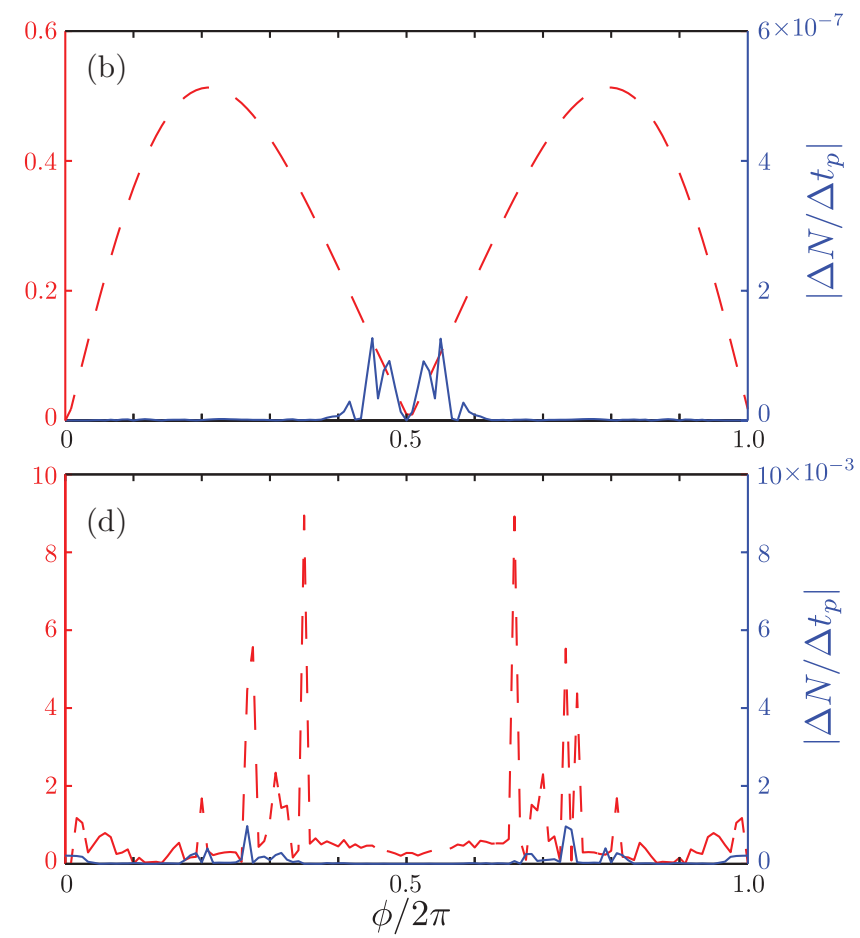

FIG. 10. (Color online) Sensitivity of the density measurements for interaction strengths of (a) and (b) $g=-0.06$ and (c) and (d) $g=0.3$ with respect to changes in phase and total pulse time. The dashed lines correspond to the phase sensitivity $\partial N / \partial \phi$, and the solid lines correspond to the pulse-time sensitivity $\partial N / \partial t_{p}$ between $90 \Omega^{-1} \leqslant t_{p} \leqslant 110 \Omega^{-1}$ for (a) and (c) and $1800 \Omega^{-1} \leqslant t_{p} \leqslant 2200 \Omega^{-1}$ for (b) and (d). 


\section{CONCLUSION}

We have demonstrated that the f-CTAP protocol is a valid methodology for the coherent spatial splitting of a BEC in the presence of interactions. Reversal of the f-CTAP protocol provides a robust phase-sensitive measurement as an alternative to traditional methods. This interferometric process is robust to changes in pulse time once in the adiabatic regime for the small interaction limit. Also, even though the adiabatic principle is not strictly valid in this nonlinear system, it is possible to find splitting and recombination time scales that achieve the balance between maintaining the system in the $D_{0}^{\prime}$ stationary state and divergence due to instability. Phase-sensitive measurements in the presence of nonlinear atomic interactions can lead to an enhancement in sensitivity without significant loss in robustness with respect to changes in pulse time.

\section{ACKNOWLEDGMENTS}

The authors thank C. Hill for helpful discussions. A.D.G. acknowledges the Australian Research Council for financial support (Project No. DP0880466).
[1] M. H. Anderson, J. R. Ensher, M. R. Matthews, C. E. Wieman, and E. A. Cornell, Science 269, 198 (1995).

[2] K. B. Davis, M.-O. Mewes, M. R. Andrews, N. J. van Druten, D. S. Durfee, D. M. Kurn, and W. Ketterle, Phys. Rev. Lett. 75, 3969 (1995).

[3] S. Raghavan, A. Smerzi, S. Fantoni, and S. R. Shenoy, Phys. Rev. A 59, 620 (1999).

[4] Y. P. Huang and M. G. Moore, Phys. Rev. A 73, 023606 (2006).

[5] M. Lewenstein, A. Sanpera, V. Ahufinger, B. Damski, A. Sen De, and U. Sen, Adv. Phys. 56, 243 (2007).

[6] I. Bloch, J. Dalibard, and W. Zwerger, Rev. Mod. Phys. 80, 885 (2008).

[7] Y.-J. Wang, D. Z. Anderson, V. M. Bright, E. A. Cornell, Q. Diot, T. Kishimoto, M. Prentiss, R. A. Saravanan, S. R. Segal, and S. Wu, Phys. Rev. Lett. 94, 090405 (2005).

[8] T. Schumm, S. Hofferberth, L. M. Andersson, S. Wildermuth, S. Groth, I. Bar-Joseph, J. Schmiedmayer, and P. Krüger, Nat. Phys. 1, 57 (2005).

[9] Y. Shin, M. Saba, T. A. Pasquini, W. Ketterle, D. E. Pritchard, and A. E. Leanhardt, Phys. Rev. Lett. 92, 050405 (2004).

[10] Y. Castin and J. Dalibard, Phys. Rev. A 55, 4330 (1997).

[11] G.-B. Jo, J.-H. Choi, C. A. Christensen, T. A. Pasquini, Y.-R. Lee, W. Ketterle, and D. E. Pritchard, Phys. Rev. Lett. 98, 180401 (2007).

[12] W. Li, A. K. Tuchman, H.-C. Chien, and M. A. Kasevich, Phys. Rev. Lett. 98, 040402 (2007).

[13] P. Marte, P. Zoller, and J. L. Hall, Phys. Rev. A 44, R4118 (1991).

[14] J. Oreg, F. T. Hioe, and J. H. Eberly, Phys. Rev. A 29, 690 (1984).

[15] J. R. Kuklinski, U. Gaubatz, F. T. Hioe, and K. Bergmann, Phys. Rev. A 40, 6741 (1989).

[16] U. Gaubatz, P. Rudecki, S. Schiemann, and K. Bergmann, J. Chem. Phys. 92, 5363 (1990).

[17] K. Bergmann, H. Theuer, and B. W. Shore, Rev. Mod. Phys. 70, 1003 (1998).

[18] J. Lawall and M. Prentiss, Phys. Rev. Lett. 72, 993 (1994).

[19] M. Weitz, B. C. Young, and S. Chu, Phys. Rev. A 50, 2438 (1994).

[20] M. Weitz, B. C. Young, and S. Chu, Phys. Rev. Lett. 73, 2563 (1994).

[21] K. Eckert, M. Lewenstein, R. Corbalán, G. Birkl, W. Ertmer, and J. Mompart, Phys. Rev. A 70, 023606 (2004).

[22] A. D. Greentree, J. H. Cole, A. R. Hamilton, and L. C. L. Hollenberg, Phys. Rev. B 70, 235317 (2004).
[23] C. J. Bradly, M. Rab, A. D. Greentree, and A. M. Martin, Phys. Rev. A 85, 053609 (2012).

[24] V. O. Nesterenko, A. N. Novikov, F. F. Souza Cruz, and E. L. Lapolli, Laser Phys. 19, 616 (2009).

[25] B. Liu, L.-B. Fu, S.-P. Yang, and J. Liu, Phys. Rev. A 75, 033601 (2007).

[26] G.-F. Wang, D.-F. Ye, L.-B. Fu, X.-Z. Chen, and J. Liu, Phys. Rev. A 74, 033414 (2006).

[27] A. P. Itin and S. Watanabe, Phys. Rev. Lett. 99, 223903 (2007).

[28] E. M. Graefe, H. J. Korsch, and D. Witthaut, Phys. Rev. A 73, 013617 (2006).

[29] M. Rab, J. H. Cole, N. G. Parker, A. D. Greentree, L. C. L. Hollenberg, and A. M. Martin, Phys. Rev. A 77, 061602(R) (2008).

[30] B. O'Sullivan, P. Morrissey, T. Morgan, and T. Busch, Phys. Scr. T140, 014029 (2010).

[31] F. Dreisow, M. Ornigotti, A. Szameit, M. Heinrich, R. Keil, S. Nolte, A. Tunnermann, and S. Longhi, Appl. Phys. Lett. 95, 1102 (2009).

[32] R. Menchon-Enrich, A. Llobera, V. J. Cadarso, J. Mompart, and V. Ahufinger, IEEE Photonics Technol. Lett. 24, 536 (2012).

[33] S. Hofferberth, I. Lesanovsky, B. Fischer, J. Verdu, and J. Schmiedmayer, Nat. Phys. 2, 710 (2006).

[34] S. Zhang and F. Wang, Phys. Lett. A 279, 231 (2001).

[35] R. Franzosi and V. Penna, Phys. Rev. E 67, 046227 (2003).

[36] C. Ottaviani, V. Ahufinger, R. Corbalán, and J. Mompart, Phys. Rev. A 81, 043621 (2010).

[37] A. Smerzi, S. Fantoni, S. Giovanazzi, and S. R. Shenoy, Phys. Rev. Lett. 79, 4950 (1997).

[38] S. Burger, K. Bongs, S. Dettmer, W. Ertmer, K. Sengstock, A. Sanpera, G. V. Shlyapnikov, and M. Lewenstein, Phys. Rev. Lett. 83, 5198 (1999).

[39] L. Khaykovich, F. Schreck, G. Ferrari, T. Bourdel, J. Cubizolles, L. D. Carr, Y. Castin, and C. Salomon, Science 296, 1290 (2002).

[40] K. E. Strecker, G. B. Partridge, A. G. Truscott, and R. G. Hulet, Nature (London) 417, 150 (2002).

[41] S. L. Cornish, S. T. Thompson, and C. E. Wieman, Phys. Rev. Lett. 96, 170401 (2006).

[42] O. Romero-Isart, K. Eckert, C. Rodó, and A. Sanpera, J. Phys. A: Math. Theor. 40, 8019 (2007).

[43] K. Nemoto, C. A. Holmes, G. J. Milburn, and W. J. Munro, Phys. Rev. A 63, 013604 (2000). 
[44] Q. Thommen, J. C. Garreau, and V. Zehnlé, Phys. Rev. Lett. 91, 210405 (2003).

[45] R. D’Agosta and C. Presilla, Phys. Rev. A 65, 043609 (2002).

[46] H. Goldstein, C. P. Poole, Jr., and J. L. Safko, Classical Mechanics, 3rd. ed. (Addison-Wesley, Reading, MA, 2001).
[47] M. Fattori, G. Roati, B. Deissler, C. D’Errico, M. Zaccanti, M. Jona-Lasinio, L. Santos, M. Inguscio, and G. Modugno, Phys. Rev. Lett. 101, 190405 (2008).

[48] K. Winkler, F. Lang, G. Thalhammer, P. v. d. Straten, R. Grimm, and J. H. Denschlag, Phys. Rev. Lett. 98, 043201 (2007). 